\title{
Relación entre la resistencia a compresión de probetas-testigo y probetas normalizadas
}

\author{
MARIANO PÉREZ SÁNCHEZ, Lcdo. en Ciencias Químicas. Laboratorio Central de Materiales de la \\ Confederación Hidrográfica del Duero. VALLADOLW
}

Fecha de recepción: $8-\mathrm{V}-97$

Fecha de aceptación: 22-VII-97

ESPAÑA

\section{RESUMEN}

Los criterios relativos a la Resistencia a Compresión de un hormigón obtenida sobre especimenes NORMALIZADOS y EXTRAIDOS (TESTIGOS) han sido expuestos y tratados por diversos autores en varias de sus obras de forma aislada y sin que exista un compendio que los atiendci en su conjunto; por ello pretendemos aquí su concatenación, no sólo exponiéndolos sino también tratando de explicar abreviadamente los fenómenos que los implican, sobre todo en aquellos casos en los que la bibliografía no lo hace o bien lo hace de modo insuficiente para el no experto.

Nuestra contribución al tema se limita, pues, a un ejercicio de compilación de los conocimientos actuales y a una modesta y resumida exposición de consideraciones propias.
SUMMARY

Criteria about concrete Strength Resistance of DRILLED STANDARDIZED specimens have been considered and occasionally explained by several authors in some of their work, but there is no publication devoted to the full extent of the subject., reason why we are trying to put together resulis/ideas, expose them, and briefly explain all phenomena implied, specially in those cases where bibliography does not satisfy the needs of the non-expert.

Our contribution limits itself to a compilation work of the present knowledge and a modest and abridged exposé of our own considerations.

\section{INTRODUCCIÓN}

La "Instrucción para el Proyecto y Ejecución e Obras de Hormigón en Masa o Armado, EH- 91" (1) especifica, en su Articulo $66^{\circ}$, que la Resistencia a Compresión de un Hormigón (Rc) es la que se obtiene sobre Probetas Normalizadas ( $P N$ ), es decir, cilíndricas de $15 \mathrm{~cm}$ de diámetro y $30 \mathrm{~cm}$ de altura (Esbeltez $\mathrm{E}=2$ ) y ensayadas a los 28 dias de edad, tal como prescriben las Normas UNE (2); ello supone que tales probetas se confeccionarán, conservarán y romperán a compresión en condiciones estandarizadas lo que implica, entre otras consideraciones, que en el momento de su cusayo estén prácticamente saturadas de agua.
Estos comentarios preliminares tienen por objeto definir algunos aspectos fundamentales que servirán como base para establecer los criterios que relacionan la Resistencia a Compresión de un hormigón (Re) obtenida sobre Probetas Testigo (PT) con la obtenida sobre Probetas Normalizadas (PN) o relación Rethen.

Por lo ya expuesto, es evidente que trataremos, exclusivamente, de especimenes CILINDRICOS.

\section{FACTORES DE CORRCCCION}

Querenos insistir en el hecho de que ol objetivo aquí perseguido es tratar de estabiccer la relación entre ken y 
Ret mediante la aplicación, a estas últimas, de los correspondientes Factores de Corrección (Fx).

En este sentido, se comprende que el hormigón constituyente de una PT no ha seguido el mismo proceso evolutivo que el de una PN, ni la obtención de ambas es semejante, por lo que sus respectivas Rc no son, en principio, comparables; para que lo sean deberán de aplicarse a las Rct los referidos FX de donde resultará:

$$
\text { Rct. Ft }=\text { Ren }
$$

siendo:

$$
\begin{aligned}
& \text { Ret : } \text { Resistencia a Compresión de la PT } \\
& \text { Ren : Id. Id. Id PN } \\
& \text { Ft : Factor de Correc. Total = F1 } \cdot \text { F2 }
\end{aligned}
$$

Las magnitudes de los Fx de los que vamos a tratar se derivan siempre de datos obtenidos experimentalmente y son, por tanto, variables, dentro de un intervalo más o menos amplio, por lo que el atribuirles valores concretos es un ejercicio al menos atrevido, dado el cúmulo de circunstancias que los afectan; aunque aquí lo haremos, será con carácter puramente indicativo en pro de la simplificación.

En la bibliografía relacionada pueden encontrarse explicaciones más detalladas sobre las particularidades y fenómenos implicados en cuanto a la adopción de tales Fx, puesto que aquí nos limitaremos a exponerlos resumidamente.

A nuestro juicio, las obras de M. F. Cánovas son las que comentan de forma más completa e instructiva la serie de circunstancias a considerar sobre el tema y es por ello que las hemos tomado (siempre que ha sido posible) como principal referencia cuando no existe normativa UNE al respecto, aunque sin obviar en ningún momento los estudios y conclusiones de los demás autores incluidos en la bibliografía reseñada; por tal motivo, cuando en el texto no se concreta cita bibliográfica respecto a una determinada influencia, debe sobrentenderse comentada en las obras del citado profesor $(7,8)$.

\subsection{Por condiciones de ejecución normalizada (F1)}

Es patente que las circunstancias inherentes a las PN (ejecución, homogeneidad, curado, etc.), son francamente favorables respecto a las del mismo hormigón colocado en obra; como consecuencia, las PT extraídas de éste darán una $R \mathbf{c}$ más baja que la correspondiente a aquellas. Por tal motivo, la "Comisión Permanente del Hormigón" (C.P.H.) y, en general, la bibliografía consultada, recomiendan exigir a las PT sólo el $\mathbf{9 0 \%}$ de la Rc de las PN.
El profesor M.F. Cánovas $(7,8)$ es más exhaustivo respecto a este Factor de Corrección F1 puesto que io relaciona con la Rc del hormigón implicado, de tal forma que la correspondencia aproximada sería:

Rc del hormigón implicado (kp/ $\left.\mathrm{cm}^{2}\right): 200 \quad 300 \quad 400$ Factor Correcc. F1: $1,051,151,30$

y cabe suponer que, de forma razonable, tales valores puedan ser objeto de interpolación y extrapolación.

Pero dado que la "C.P.H." recomienda el valor $\mathbf{F 1}=1,1$, lo aceptamos con las reservas que apunta el citado autor en el sentido de considerarlo excesivamente conservador; por tanto adoptaremos:

$$
F 1=1,1
$$

\subsection{Por condiciones intrínsecas ( F2 )}

En este apartado cabe considerar todas aquellas particularidades inherentes a las PT, a las que denominaremos INTRÍNSECAS a ellas, y que influyen en la relación de su Rc respecto a la de las probetas MOLDEADAS (PM) y, consecuentemente, a las de las PN puesto que éstas también lo son.

En general, los autores consultados cuando hacen referencia a ellas las tratan de forma aislada, sin proponer una magnitud $\mathrm{F} 2$ que las englobe; yes evidente la dificultad de hacerlo, dada su diversidad.

Así, autores como R. H. Cambel y R.E. Robins (3) consideran comprobado que, en igualdad de otras condiciones, la Rc de una PT es del orden del $10 \%$ menor que la de la correspondiente PM, atribuyéndolo, esencialmente, a la microfisuración interna que produce la sonda de extracción en la PT; es, pues, un ejemplo de considerar aisladamente una influencia (microfisuración) $o$, al menos, de significarla en detrimento de otras que, aunque no mencionadas, comparten con ella esa minoración del $10 \%$.

Es en la obra del profesor M. F. Cánovas $(7,8)$ donde encontramos referencia, más o menos dispersa, a todas ellas, procurándonos, así, una base para intentar extraer finalmente una conclusión condicionada.

Por razones instructivas, relacionamos resumidamente las más significativas, a nuestro juicio, indicando el sentido de su implicación en la PT.

\section{Influencias positivas}

Efecto Pared: en toda PM se produce una acumulación de pasta y mortero de cemento entre las paredes del molde y 
el esqueleto pétreo del espécimen lo que, evidentemente, no ocurre en una PT; la mayor debilidad de esas zonas en la PM se traduce en una superior Rc de la PT, supuestas ambas sometidas a idénticas condiciones de curado y climatológicas.

\section{Influencias negativas}

Microfisuración: ya comentada anteriormente, supone un debilitamiento de la PT y su influencia es tanto más acentuada cuanto menor es su diámetro.

Condiciones de Contorno: irregularidades en la superficie lateral de la PT, provocadas, asimismo, por la sonda de extracción y que reducen su Rc.

Relación entre diámetro de la PT y Tam. Máx. de Árido: está también comprobado que la sonda de extracción afecta negativamente a la Rc de la PT tanto más cuanto menor es su diámetro en relación al tamaño máximo del árido incluido; para paliarlo, algunas normas, entre ellas la UNE-83.302/84 indican que el diámetro de la PT conviene sea $>$ tres veces dicho tamaño máximo y en ningún caso inferior a 2 .

En la obra del profesor M.F. Cánovas (8), queremos intuir que, atenuadas al máximo las influencias descritas, se acepta la recomendación de algunas normas en el sentido de que la Rc de la PT es entonces del orden del $10 \%$ al $15 \%$ menor que la de la PM, y por extensión, que la de la PN; por tanto, tratando de llegar a una simplificación del tema, adoptaremos como magnitud del conjunto de tales influencias (o CONDICIONESINTRÍNSECAS), aplicable a la Rc de la PT la media de ambos valores límite:

$$
\mathrm{F} 2=1,125
$$

Creemos importante añadir que las PT no deben extraerse antes de los 14 días de edad del hormigón por el peligrode su excesivo debilitamiento si la Rc del hormigón es $<50 \mathrm{kp} / \mathrm{cm}^{2}$, procurándolas, además, en las zonas centrales del elemento a fin de evitar las consecuencias del Efecto Pared que se producirá en las laterales, por su proximidad a las superficies encofradas $(7,8)$

\subsection{Por esbeltez normalizada ( F3 )}

Las normativas actuales refieren la Rc del hormigón al ensayo sobre especímenes de altura doble que el diámetro $(\mathbf{E}=2)$ ya que este parámetro influye decisivamente en el valor obtenido para la Rc en el sentido de que, a igualdad de condiciones restantes, disminuye cuando $\mathbf{E}$ aumenta y viceversa; la normativa española (UNE-83.302/84) especifica claramente la obligatoriedad de que $\mathbf{E}=\mathbf{2}$.

Conviene aclarar que para una mejor comprensión de los razonamientos a exponer, las consideraciones sobre la influencia de E respectoa Rc las referiremos a especímenes de igual DIÁMETRO; precisado esto, tal influencia se relaciona esencialmente con:

Efecto Pandeo: damos por conocido el hecho de sus connotaciones negativas en relación a la Rc del elemento implicado, en el sentido de que $\mathbf{E}$ y $\mathbf{R}$ c están en relación inversa.

Efecto Volumen: cuanto mayor es el volumen del elemento mayor es también su heterogeneidad $y$, consecuentemente, la preponderancia de las zonas débiles del mismo; Albert Joisel (4) lo expone en un elaborado estudio teórico sobre el tema llegando a la conclusión compartida por otros diversos autores $(5,6,7,8)$ de que a mayor volumen del espécimen ensayado corresponderá menor Rc y recíprocamente.

Puesto que a igualdad de diámetro y mayor esbeltez corresponde mayor volumen del espécimen es obvio que le serán aplicables las conclusiones anteriores.

Efecto Zunchado: el rozamiento con los platos de la prensa da lugar a un confinamiento de las cabezas del espécimen (zunchado) que se traduce en un tipo de rotura cónica cuyas bases con dichas cabezas. El fenómeno puede paliarse mediante interposición de ciertos artilugios entre ambos e incluso con modificaciones de las superficies de contacto de los platos; pero en definitiva, si se produce el efecto en cuestión suponemos conocido el hecho de que su influencia respecto a la $\mathbf{R} \mathbf{c}$ del espécimen está en razón inversa a su esbeltez.

Cada uno de lo efectos considerados (y por tanto su resultante) apuntan en el sentido de poder resumir que Rc y $\mathbf{E}$ están siempre en relación inversa y aunque los distintos autores concuerdan en ello difieren sin embargo, en cierta medida, al acotar los valores de tal relación; pero en este caso concreto eludimos esas discrepancias puesto que la normativa española, previsiblemente en base a los efectos comentados y a su potencial influencia, dispone de la norma UNE-83.302/84 al respecto y es lógico que adoptemos las magnitudes que en ella se indican y que transcribimos a continuación (Tabla 1).

TABLA 1

\begin{tabular}{|c|c|}
\hline HSB HWYVL & HATOH SORHEQ \\
\hline 2,00 & 1,00 \\
\hline 1,75 & 0,98 \\
\hline 1,50 & 0,96 \\
\hline 1,25 & 0,94 \\
\hline 1,10 & 0,90 \\
\hline
\end{tabular}


Según la citada Norma, el valor mínimo deberá ser $\mathbf{E}=1,00$ y los valores hasta $\mathbf{E}=2$ pueden calcularse por interpolación.

\subsection{Por dimensiones normalizadas ( F4 )}

Anteriormente hemos razonadola introducción del Factor de Corrección F3 como necesario para referir las Rc a especímenes de $\mathbf{E}=\mathbf{2}$ y para una más fluida comprensión de las consideraciones a exponer, las relacionábamos con la condición de igual diámetro.

Pero para especímenes de $\mathbf{E}=\mathbf{2}$ y distinto diámetro, evidentemente resultarán distintos VOLÚMENES y ya se ha indicado en el Apartado 2.3 la influencia de este parámetro sobre Rc; el Factor de Corrección F4 del que ahora tratamos tiene como fundamento corregir la Rc de especímenes que siendo su $\mathbf{E}=\mathbf{2}$ tengan, sin embargo, distinto volumen $y$, en definitiva, distintas DIMENSIONES; es evidente, por tanto, que el DIÁMETRO será, en este caso, la magnitud clave de influencia y su variación conlleva, principalmente:

Efecto Volumen: un incremento positivo o negativo del diámetro del espécimen se traduce en una modificación proporcional de su volumen y, por tanto, de su Rc tal como queda expuesto en $\mathbf{2 . 3}$.

Efecto Zunchado: en 2.3 se explican las causas de su origen y relación con $\mathbf{R c}$ y $\mathbf{E}$; pero también es conocida su influencia respecto al diámetro del espécimen en cuanto a su relación inversa con $\mathbf{R c}$.

Al ser del mismo sentido ambos efectos lo será también su resultante; se concluye, por tanto, la relación inversa entre el DIÁMETRO del espécimen y su Rc y como consecuencia última, de sus DIMENSIONES y Rc puesto que estamos tratando con especímenes de la misma esbeltez $(\mathbf{E}=2)$.

Dado que el objetivo que nos proponemos es el referir $\mathbf{R c}$ de PT a Rc de PN y siendo $15 \mathrm{~cm}$ de diámetro y $30 \mathrm{~cm}$ de altura las DIMENSIONES NORMALIZADAS (Apartado 1), el Factor de Corrección F4 correspondiente queda acotado en el Cuadro "10.4.a" de la EH-91 que, lógicamente, adoptamos, pero ampliado con los datos del Cuadro "10.3" que el profesor M. F. Cánovas incluye en su obra (8), y que resumimos en la tabla 2.

Aunque nada se indica al respecto se intuye que para otras dimensiones de especímenes (siempre de $\mathbf{E}=2$ ) cabe obtener el correspondiente F4 por interpolación o extrapolación, dentro de límites prudenciales.

\subsection{Por edad normalizada ( F5 )}

Es sabido que la Rc del hormigón varía con su EDAD en el sentido de que, salvo casos paradógicos aislados que tienen su particular explicación, ambas están en razón directa; por tanto a edades distintas corresponderán diferentes Rc.

Siendo 28 días la EDAD NORMALIZADA (Apartado 1), el Factor de Corrección F5 implicado será el que corresponda a los valores específicados en el Cuadro "10.4.b" de la EH-91 (1), el cual tomamos como referencia en la tabla 3.

Como anteriormente (Apartado 2.4) se intuye la posibilidad de interpolación y extrapolación.

TABLA 2

\begin{tabular}{||c|c|c|}
\hline $\begin{array}{c}\text { DIMENSIONES DEL ESPÉCIMEN } \\
\text { (cm) }\end{array}$ & LIMITES DE VARIACIÓN \\
DE F4 & $\begin{array}{c}\text { VALOR MEDIO } \\
\text { DE F4 }\end{array}$ \\
\hline $10 \times 20$ & 0,94 a 1,00 & 0,97 \\
$15 \times 30$ & No se indican & 1,00 \\
$20 \times 40$ & 1,00 a 1,10 & 1,03 \\
$25 \times 50$ & No se indican & 1,05 \\
$30 \times 60$ & 1,10 \\
\hline
\end{tabular}

TABLA 3

\begin{tabular}{|c|c|c|c|c|c|}
\hline \multirow{2}{*}{$\begin{array}{c}\text { FACTOR DE CORRECCIÓN F'5 } \\
\text { PARA HORMIGONES. }\end{array}$} & \multicolumn{5}{|c|}{ EDAD DEL HORMIGÓN EN DÍAS. } \\
\cline { 2 - 6 } & 3 & 7 & 28 & 90 & 360 \\
\hline Endurecimiento Normal & 0,40 & 0,65 & 1,00 & 1,20 & 1,35 \\
\hline Endurecimiento Rápido & 0,55 & 0,75 & 1,00 & 1,15 & 1,20 \\
\hline
\end{tabular}


Como puede observarse, los valores de la Tabla 3 son de aplicación al espécimen de EDAD NORMALIZADA (28 días ) para referir su $\mathbf{R c}$ a otras edades; como en nuestra proposición (Apartado 2) todos los Factores de Corrección (Fx) son aplicables a la Rc de la PT, es obvio que:

$$
\text { F5 }=1 / F^{\prime} 5
$$

Reiteramos (Apartado 2.2) que la extracción de la PT no debe de realizarse sobre hormigones de edad inferior a 14 días.

\subsection{Por estado de saturación normalizada (F6)}

El agua infiltrada en el espécimen puede actuar como lubrificante interno, minorando, así, su Rc; además $(7,8,9,10,11)$ al ser absorbida por los geles de cemento parece ser que los debilita disminuyendo las fuerzas de cohesión entre las partículas sólidas.

En definitiva, los estudios experimentales demuestran que la Rc de un espécimen varía en sentido inverso de su grado de saturación $(8,9)$.

Puesto que la meta que aquí nos proponemos es relacionar Rc de PT con las de PN considerandoa estas últimas como SATURADAS (Apartado 1) y siendo la Rc de la PT variable según su grado de saturación, parece evidente que si queremos concretar un Factor de Corrección F6 al respecto tengamos que considerar a la PT en condiciones bien definidas, esto es, en estado SATURADO o en estado SECO, entendiendo como tal al que corresponde a una exposición en ambiente de Humedad Relativa dentro de los límites aproximados del $45 \%$ y $60 \%$ (10) que es el, por lo general, dominante en los laboratorios de ensayo.

Bajo esta proposición es obvio que si consideramos a la PT como SATURADA, el F6 que la relaciona con la PN será la unidad; si por el contrario, la suponemos SECA, adoptaremos como valor medio $\mathrm{F} 6=1 / 1,15=0,87(7,8)$.

Por tanto, la magnitud a aplicar a la Rc de la PT para relacionarla con la correspondiente de la $\mathbf{P N}$ sería:

$\begin{array}{ll}\text { F6 }=: 1 & \text { (Para PT en estado SATURADO) } \\ \text { F6 }=0,87 & \text { (Para PT en estado SECO) }\end{array}$

$\mathrm{Y}$ para grados de saturación intermedios consultar la bibliografía de referencia.

\subsection{Por influencia de barras / armaduras ( F7)}

No se aprecia prácticamente influencia en la $\mathbf{R} c$ de la $\mathbf{P T}$ por el hecho de que incluya barras transversales de armadura oporque se haya extraído en zona en queésta sea densa $(7,8,9,10)$ siempre que tales circunstancias no sobrepasen los límites que indican los repetidos autores, incluyendo el de no contener barras cuyo eje coincida con el de la PT; en caso de que la PT no cumpla tales requisitos deberá rechazarse por inaceptable.

Por tanto se desprende que el potencial Factor de Corrección correspondiente (F7) se puede obviar a todos los efectos puesto que, en todo caso, sería:

$$
\mathbf{F 7}=1
$$

\subsection{Factores de corrección de aplicación restringida (F8.1 y F8.2)}

Los autores M. F. Cánovas y A. Delibes $(7,8,10)$ exponen respecto a las PT una serie de circunstancias que pueden afectar a su Rc; pero, en tales casos, los correspondientes Factores de Corrección Fx son de aplicación exclusiva entre ellas, en base a decidir cuál ha de ser la Rc elegida para relacionar con la de la PN. Por tal exclusividad los definimos aquí como de aplicación RESTRINGIDA a las PT entre sí; los resumimos a continuación, pero remitiéndonos siempre a la bibliografía reseñada para cualquier aclaración sobre el tema:

\subsubsection{Por influencia de la direcc. extracción (F8.1)}

Independientemente de la zona considerada, si una PT se extrae perpendicular a la dirección del hormigonado (por logeneral, realizada en sentido vertical) se obtiene una Rc menor que si se extrae paralelamente a ella, hecho que ocurre, inevitablemente, en pilares. Parece evidente (como causa del fenómeno) que la distinta dificultad de efectuar la extracción en ambos casos ha de afectar en grado diferente a la PT (10), pero también se desprende $(7,8)$ que la extracción perpendicular al hormigonado ejerce su influencia en el sentido de que modifica relativamente la constitución interna del espécimen; si esta última fuese la influencia preponderante, la $\mathbf{R} \mathbf{c}$ a relacionar con la de la PN debería ser la de la PT extraída en la dirección del hormigonado, por similitud de ejecución en ambos casos (PN y hormigonado).

Pero se comprende que, al limitarse esta circunstancia exclusivamente a la relación de las $\mathbf{R c}$ entre PT, deberá de ser el técnico responsable quien tome la decisión de elegir cuál de ellas hará corresponder con la de la PN.

En definitiva, según los autores citados, la diferencia en cuestión puede estimarse del orden del $5 \%$ por lo que siendo:

$\mathbf{R c t p}=\mathbf{R c}$ de la $\mathbf{P T}$ extraída en dirección PERPENDICULAR a la de hormigonado. 


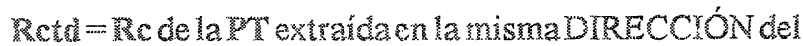
hormigonado.

T8.1 - Eactor de Corrección aplicable a Rcrp $=1,05$

resultaría:

$$
\begin{aligned}
& \text { Rctd }=78.1 \quad \text { Retp } \\
& \text { Retd }=1,05 \quad \text { Retp }
\end{aligned}
$$

\subsubsection{Por influencia de la zona deextracción (F8.2)}

Estudios experimentales demuestran que en las zonas relativamente próximas a la superior de elementos hormigonados verticalmente, la Rc del hormigón es menor que en las inferiores; aunque su puesta en obra sea correcta, fenómenos tales como exudación, segregación, incremento de densidad con la profundidad como consecuencia del propio peso acumulado, etc., se aceptan como causa fundamental de ello.

En los cuadros 1 y 2 del gráfico 1 resumimos las magnitudes propuestas por ambos autores para el Factor de Corrección a considerar (F8.2) según la zona de extracción de la $P T$; se desprende la mayor precisión del cuadro 1 , aunque no acota valores cuando resulta $\mathrm{H}<2,00 \mathrm{~m}$.

Como en el caso anterior (F8.1), la aplicación del Factor de Corrección $\mathbf{1 8 . 2}$ se reduce a la relación de las $\mathrm{Rc}$ de la PT entre sí y el técnico experto será quien determine no sólo su magnitud sino también la Rc de la PT que opta por considerar para su posterior relación con la de la $\mathrm{PN}$.

En lo que sigue damos por supuesto que la Reti de los cuadros 1 y 2 ha sido ya corregida por el Factor de Corrección 8.1 , anteriormente tratado.

Gráfico. 1

FACTOR DE CORRECCIÓN F8.2 SEGÚN:

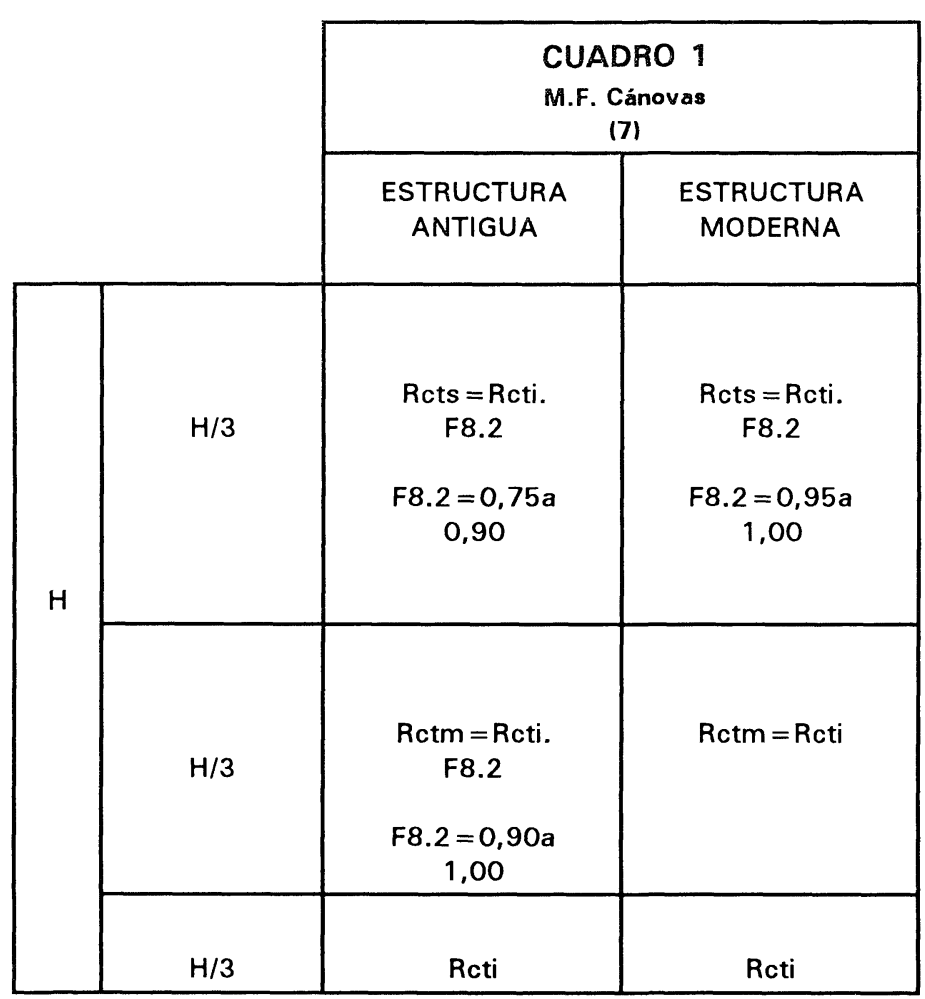

$H \geq 2,00 m$

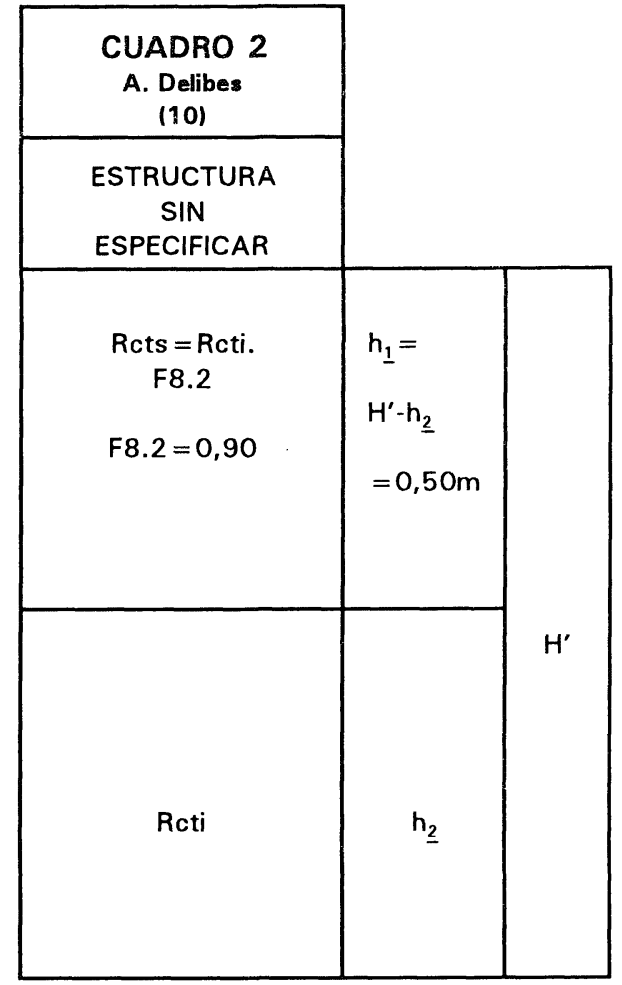

$H^{\prime}=h_{1}+h_{2}=\sin$ especificar

Siendo:

Rcts = Rc de la PT extraída en la zona SUPERIOR; $\mathbf{R c t m}=$ Id. id. zona MEDIA

Rcti $=$ Id. id. zona INFERIOR 
Como complemento al Cuadro 1, el profesor M. F. Cánovas (7) indica que, en base a la experiencia actual, las diferencias entre las distintas Rct desaparecen, prácticamente, para hormigones de alta resistencia $\left(\boldsymbol{R c}>500 \mathrm{kp} / \mathrm{cm}^{2}\right.$ ), siendo, entonces $\mathbf{F 8 . 2}=1 ; \mathrm{y}$, aunque no lo especifica, suponemos que, en pro de la seguridad, se refiere al caso de que Rcts $>500 \mathrm{kp} / \mathrm{cm}^{2}$.

\subsection{Resumen sobre los factores de corrección Fx}

Como ya quedó indicado en el Apartado 2, las magnitudes de todos los Fx son de aplicación a las Rc de PT (o Rct) a efectos de referirlas a las Rc de PN (o Rcn); las denominaciones que les hemos atribuido tratan de referirse a los condicionamientos de su implicación:

F1 a F7 :de aplicación DIRECTA a las Rct, para su correspondencia con las $\mathbf{R c n}$.
F1 y F2 :de aplicación OBLIGADA a las Rct, porque influyen siempre en su relación con las $R \mathbf{R e n}$.

F3 a F7 :de aplicación CIRCUNSTANCIAL a las Rct, porque su utilización no siempre es necesaria para relacionarlas con las $\mathbf{R c n}$.

F8.1 y F8.2 :de aplicación RESTRINGIDA a las Rct, entre sí, para elegir la que se relacionará con la $\mathbf{R} \mathbf{c n}$

Acorde con lo anterior, les corresponderán las denominaciones:

F1 y F2: de aplicación DIRECTA y OBLIGADA.

F3 a F7: deaplicación DIRECTA Y CIRCUNST ANCIAL.

F8.1 y F8.2 :de aplicación RESTRINGIDA.

CUADRO-RESUMEN de los Fx tratados y las consideraciones expuestas sobre ellos.

A continuación, incluimos un CUADRO-RESUMEN sobre todos los Fx tratados y las consideraciones expuestas sobre ellos

\begin{tabular}{|c|c|c|c|c|c|}
\hline \multicolumn{6}{|c|}{ FACTORES DE CORRECCION. CUADRO RESUMEN } \\
\hline $\begin{array}{l}\text { Denomi- } \\
\text { nación }\end{array}$ & Valor & $\begin{array}{l}\text { Tipo de } \\
\text { Aplicación }\end{array}$ & $\begin{array}{l}\text { Para } \\
\text { Relacionar } \\
\text { AR }\end{array}$ & Objeto de la Aplicación & $\begin{array}{l}\text { Ver } \\
\text { Aparta- } \\
\text { do }\end{array}$ \\
\hline F1 & 1,1 & Directa-Obligada & $\begin{array}{l}\text { Ret con } \\
\text { Ren }\end{array}$ & $\begin{array}{l}\text { Para referir a Condiciones de } \\
\text { Ejecución Normalizadas. }\end{array}$ & 2.1 \\
\hline F2 & 1,125 & Id. & Id. & $\begin{array}{c}\text { Para compensar Condiciones } \\
\text { Intrínsecas. }\end{array}$ & 2.2 \\
\hline F3 & Tabla. 1 & $\begin{array}{l}\text { Directa- } \\
\text { Circunstancial }\end{array}$ & Id. & $\begin{array}{c}\text { Para referir a Esbeltez } \\
\text { Normalizada. }\end{array}$ & 2.3 \\
\hline F4 & Id. 2 & Id & Id. & $\begin{array}{l}\text { Para referir a Dimensiones } \\
\text { Normalizadas. }\end{array}$ & 2.4 \\
\hline F5 & Id. 3 & Id & Id & Para referir a Edad Normalizada & 2.5 \\
\hline F6 & $\begin{array}{c}\text { (a) } \\
1 \\
0,87\end{array}$ & Id. & Id. & $\begin{array}{c}\text { Para referir a Saturación } \\
\text { Normalizada. }\end{array}$ & 2.6 \\
\hline F7 & (b) & Id. & Id. & $\begin{array}{c}\text { Por influencia de Barras / } \\
\text { Armaduras. }\end{array}$ & 2.7 \\
\hline F8.1 & $\begin{array}{c}\text { (c) } \\
1,05 \\
0,95\end{array}$ & Restringida & $\begin{array}{l}\text { Rct entre } \\
\text { si. }\end{array}$ & $\begin{array}{l}\text { Por efecto de Dirección de } \\
\text { Extracción. }\end{array}$ & 2.8 .1 \\
\hline F8.2 & $\begin{array}{l}\text { Gráfico } \\
1\end{array}$ & Id & Id. & $\begin{array}{l}\text { Por efecto de la Zona de } \\
\text { Extracción. }\end{array}$ & 2.8.2. \\
\hline $\begin{array}{l}\text { (a) Según } \\
\text { (b) Se de: } \\
\text { (c.) Segúr }\end{array}$ & $\begin{array}{l}\text { se ensa } \\
\text { su aplica } \\
\text { RECCIÓn }\end{array}$ & $\begin{array}{l}\text { ado SATURADO } \\
\text { acción a conside }\end{array}$ & & & \\
\hline
\end{tabular}




\section{CONSIDERACIONES FINALES}

Anteriormente (Apartado 2) hemos comentado las razones del carácter meramente indicativo de los valores atribuidos a los Factores de Corrección Fr tratados, incidiendo en el hecho de que su cuantificación la proponíamos a efectos de una simplificación del tema; deberá de ser el técnico experto el que, con subuen criterio, decida su definitiva magnitud e, incluso, deseche su aplicación, de acuerdo con las indicaciones de la bibliografía y a su propia experiencia y conocimientos.

Reiteramos que nuestro objetivo ha sido el de exponer monográficamente el conjunto de influencias que determinan la correspondencia entre Rc de PT y PN; pero en el caso de que lo que se pretenda sea conocer la $\mathbf{R c}$ real (o más probable) del hormigón constituyente de la obra, obviando su relación con la de la PN (caso frecuente), bastará aplicar a la Rc de la PT extraída aquellos Fx que el técnico considere implicados, desechando los restantes.

Por otra parte, no es demasiado arriesgado pensar que los avances de la ciencia, tecnología y experimentación puedan hacer necesario revisar algunas de las consideraciones que nos han servido comobase parallegar alas conclusiones expuestas respecto a los Factores de Corrección Fx; tal posibilidad significa una conveniente atención $y$ seguimiento del tema.

Finalmente insistir en que los conocimientos, la experiencia y el buen criterio son elementos indispensables para llegar a conclusiones correctas; es por ello que, sobre todo en casos de la más mínima incertidumbre, las decisiones finales habrán de ser tomadas por profesionales expertos.

\section{ABREVIATURAS UTILIZADAS}

\section{PM....Probeta MOLDEADA}

PN.....Probeta NORMALIZADA (también PM)

PT.....Probeta TESTIGO (extraida)

Rc.....Resistencia a Compresión, en general

Rcn.....Rc de una PN

Rct....Rc de una PT

Rctp.....Rc de una PT extraída PERPENDICULAR a la dirección del hormigonado

Rctd.....Rc de una PT extraída en la DIRECCIÓN del hormigonado

Rcts.....Rc de una PT extraída en zona SUPERIOR
Retm....Rc de una PT extraída de una zona MEDIA

Rcti....Rc de una PT extraída en zona INFERIOR

\section{E.....Esbeltez}

C.P.H.....Comisión Permanente del Hormigón

Fx....Factor de Corrección, en general

Ft....Factor de Corrección Total o producto de todos los Fx implicados.

\section{EJEMPLO DE CÁLCULO}

Expondremos un caso supuesto en el que puedan aplicarse todos los Factores de Corrección Fx de los que hemos tratado.

“En un elemento de hormigón de $1 \mathrm{~m}^{2}$ de sección y $3 \mathrm{~m}$ de altura, cuya ejecución se realizó en sentido vertical y en una sola fase, se extrae, con sonda rotatoria, una probetatestigo cuyas dimensiones al ensayo son de $10 \mathrm{~cm}$ de diámetro y $175 \mathrm{~cm}$ de altura. La extracción se efectuó a unos $2,50 \mathrm{~m}$ bajo la superficie superior del elemento, en dirección perpendicular a la de hormigonado y a los 90 días de ejecutado (estructura moderna), rompiéndose a compresión el mismo día, sin saturación previa, obteniéndose $200 \mathrm{kp} / \mathrm{cm}^{2}$. El hormigón es del tipo de "endurecimiento normal" y se desea calcular la resistencia a compresión que correspondería a la probeta normalizada que se hubiese confeccionado en su día de tal hormigón".

E1 técnico responsable del cálculo decide:

$1^{\circ}$ : referir la $\mathbf{R c}$ obtenida $\left(200 \mathrm{kp} / \mathrm{cm}^{2}\right)$ a espécimen que hubiese sido extraído en la misma dirección del hormigonado.

Según 2.8.1 :

Rctp $=200 \mathrm{kp} / \mathrm{cm}^{2}$

F8.1 $=1,05$

Rctd $=$ F8.1. Rctp

Rctd $=1,05.200$

Rctd $=210 \mathrm{kp} / \mathrm{cm}^{2}$

$2^{\circ}$ : considerar la Rc del hormigón ubicado en la zona de los $50 \mathrm{~cm}$ superiores del elemento; resuelve, además, adoptar para el Factor de Corrección correspondiente (F8.2) el valor medio que se deduzca del cuadro 1 del Apartado 2.8.2

Según 2.8.2 :

$\left(\mathrm{La}\right.$ Rctd $=210 \mathrm{kp} / \mathrm{cm}^{2}$ antes obtenida es, pues, la Rcti a 
corregir ahora, para determinar la correspondiente Rcts que se pretende).

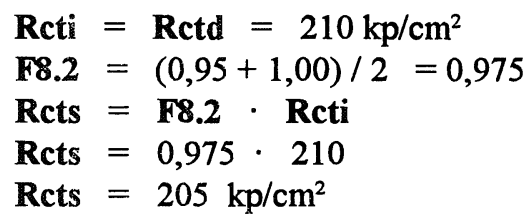

Conclusión de $1^{o}$ y $2^{o}$ :

El empleo de los Factores de Corrección de aplicación RESTRINGIDA (F8.1 y F8.2) a la Rc de la probetatestigo en cuestión (o Rctp $=200 \mathrm{kp} / \mathrm{cm}^{2}$ ) nos da el valor final de ésta si hubiese sido extraída en laDIRECCIÓN del hormigonado y en la zona de los $50 \mathrm{~cm}$ SUPERIORES del elemento, tal como decidió considerar el técnico; por tanto, la magnitud:

Rcts $=205 \mathrm{kp} / \mathrm{cm}^{2}$

es la elegida para hacerla corresponder a continuación con la de la probeta normalizada.

30. Aplicar a la Rcts elegida $\left(205 \mathrm{kp} / \mathrm{cm}^{2}\right)$ todos los Factores de Corrección de aplicación DIRECTA para referirla a la de la correspondiente probeta normalizada, adoptando como valores:

$\mathbf{F 1}=1,1$ por CONDIC. EJECUC. NORMALIZADA (2.1) F2 $=1,125$ por CONDIC. INTRÍNSECAS (2.2)
F3 $=0,98$ por ESBELTEZ NORMALIZADA (2.3)

F4 $=0,97$ por DIMENS. NORMALIZADAS (2.4)

F5 $=1 / 1,20=0,833$ por EDAD NORMALIZADA (2.5)

F6 $=0,87$ por SATURACIÓN NORMALIZADA (2.6)

F7 = --.--- no es necesaria su aplicación (2.7)

$4^{0}$. Se obtendrá así, finalmente:

Ren $=$ Rcts $\cdot$ F1 $\cdot$ F2 $\cdot$ F3 $\cdot$ F4 $\cdot$ F5 $\cdot$ F6

$\mathbf{F t}=\mathbf{F 1} \cdot \mathbf{F 2} \cdot \mathbf{F 3} \cdot \mathbf{F 4} \cdot \mathbf{F 5} \cdot \mathbf{F 6}$

$\mathbf{R c n}=\mathbf{R c t s} \cdot \mathbf{F t}$

$\operatorname{Rcn}=205 \cdot 0,853$

$\operatorname{Rcn}=175 \mathrm{kp} / \mathrm{cm}^{2}$

- Resistencia a Compresión de la PROBETA NORMALIZADA que correspondería a la de la PROBETA-TESTIGO extraída.

\section{AGRADECIMIENTOS}

Queremos significar aquí nuestro sincero agradecimiento al profesor Don Manuel Fernández Cánovas, Catedrátrico de Materiales de Construcción de la E.T.S. de Ingenieros de C.C. y P. de la Universidad Politécnica de Madrid, quien tuvo la deferencia de dedicar parte de su preciado tiempo en la revisión de este trabajo, aportando valiosas sugerencias que en él hemos incluido.

\section{BIBLIOGRAFIA}

(1) EH-91: "Instrucción para el Proyecto y la Ejecución de Obras de Hormigón en Masa o Armado".

(2) Normas UNE-83.301/84 a UNE-83.304/84.

(3) American Concrete Institute: Manual of Concrete Practice; de 4/67".

(4) ALBERT JOISEL: "Revue des Materiaux de Constructión; n 462 de 3/54"; Artículo "La Rupture du Beton en Compresión".

(5) M. VENUAT Y M. PAPADAKIS: “Control y Ensayo de Cementos, Morteros y Hormigones”; Edic. Umo; 1966.

(6) FRANCIS GORISSE: “Ensayo y Control de los Hormigones”; Editores Técnicos Asociados, S.A.; 1981.

(7) M. FDZ. CÁNOVAS: "Patología y Terapéutica del Hormigón Armado; 3 a Edición; 9/94.

(8) M. FDZ. CÁNOVAS: "Hormigón"; Ediciones 1989 y 1995.

(9) A. DELIBES LINIERS: "Tecnología y Propiedades Mecánicas del Hormigón"; Intemac; 1987.

(10) A. DELIBESLINIERS: "Análisis de la influencia de algunas variables en la Extracción y ensayo a compresión de probetas testigo de hormigón" (Separata de la Revista "Informes de la Construcción"; n 266; Publicación n 3 de 1975).

(11) J. CALAVERA: "Probetas tomadas a salida de hormigonera" (Separata de la Revista "Informes de la Construcción"; $n^{\circ} 275$; Publicación $n^{\circ} 6$ de 1975). 